\title{
Phenotypic and Functional Characterization of Lymphocytes That Bind Human Microvascular Endothelial Cells In Vitro Evidence for Preferential Binding of Natural Killer Cells
}

\author{
Jeffrey R. Bender, ** Ruggero Pardi," Marvin A. Karasek, and Edgar G. Engleman* \\ Departments of Pathology, ${ }^{*}$ Medicine (Cardiology Division), ${ }^{\ddagger}$ and Dermatology, $\$$ \\ Stanford University School of Medicine, Stanford, California 94305
}

\begin{abstract}
The microvascular endothelium has been postulated to be a critical target in the rejection of vascularized allografts. This study was undertaken to examine the ability of human sheep erythrocyte rosette forming lymphocytes (E-RFC) to form stable conjugates with microvascular endothelial cells (EC), and to assess whether a receptor-ligand interaction mediates this event. Human foreskin microvascular EC monolayers were used as targets of chromium-51-labeled E-RFC in a quantitative adherence assay. Binding was saturable, displaceable by unlabeled E-RFC, augmented by recombinant interleukin 1 (rIL-1) and inhibited by anti-LFA1 antibody. The Leu-11 + lymphocyte subset, known to be enriched for natural killer (NK) cells, bound preferentially. Only the EC-adherent lymphocyte fraction contained NK effectors, which lysed EC and classical NK targets. Thus, NK cells adhere to microvascular EC via a specific receptor-ligand interaction. The possibility exists that such binding occurs in recipients of vascularized allografts, representing the initial stage of graft rejection.
\end{abstract}

\section{Introduction}

The mechanism whereby vascularized allografts are rejected remains incompletely understood. T lymphocytes of the helper/ inducer as well as cytotoxic phenotype have been variably implicated as effectors in the rejection process $(1,2)$. Other studies have demonstrated the early localization of natural killer cells in acutely rejecting allografts (3). Additional controversy surrounds the target molecules for this process. It is generally believed that major histocompatibility antigens provide the most potent stimulus for acute rejection. However, rejection episodes can occur in HLA identical, living related donor allografts (4).

The vascular endothelium constitutes a contiguous barrier to circulating immunocompetent cells, as well as the initial site where host lymphocytes can potentially interact with major histocompatibility antigens of donor origin $(5,6)$. Endothelial cell $(E C)^{1}$ injury has been noted histologically several days before

Address reprint requests to Dr. Bender. Dr. Bender is the recipient of a National Research Service Award from the National Institutes of Health. Dr. Pardi is a postdoctoral fellow from the Ospedale S. Raffaele, Milano, Italy.

Received for publication 29 September 1986 and in revised form 3 February 1987.

1. Abbreviations used in this paper: $\mathrm{AL}$, adherent lymphocytes; $\mathrm{EC}$, endothelial cells; E-RFC, erythrocyte rosette forming cells; FACS, fluores-

J. Clin. Invest.

(C) The American Society for Clinical Investigation, Inc.

$0021-9738 / 87 / 06 / 1679 / 10 \$ 1.00$

Volume 79, June 1987, 1679-1688 functional rejection (7), with inter-EC gaps progressing to microvascular EC disintegration. It is conceivable that adhesion of lymphocytes to the endothelium may be the first step in the cascade of events leading to allograft rejection. Lymphocyte traffic into lymphoid organs appears to be regulated by binding to specialized receptors on high endothelial venules $(8,9)$. However, little is known regarding the interaction between lymphocytes and capillary endothelium outside of lymphoid organs. Human umbilical vein EC have been used as in vitro targets of lymphocyte binding $(10,11)$, and although varying degrees of lymphocyte-EC binding have been demonstrated, the specificity and molecular basis of this interaction have not been extensively studied.

In this study, we utilized monolayers of microvascular EC as targets of lymphocyte binding in an attempt to investigate whether stable lymphocyte conjugates can form in the absence of exogenous factors. The results indicate that such conjugates can indeed form and that their formation is mediated by a specific receptor-ligand interaction. Moreover, the results demonstrate a preferential and displaceable binding by natural killer (NK) cells to microvascular EC.

\section{Methods}

Microvascular EC culture. Stable cultures of human microvascular EC were established as previously described (12) with several modifications. Fresh discarded preputial skin from randomly selected anonymous newborns was initially incubated in Hanks' balanced salt solution with $\mathbf{4 0 0}$ $\mathrm{U} / \mathrm{ml}$ penicillin and $400 \mu \mathrm{g} / \mathrm{ml}$ streptomycin. The outer and inner layers were then separated with sharp dissection and the inner layer incubated for $1 \mathrm{~h}$ at $37^{\circ} \mathrm{C}$ in a $0.3 \%$ trypsin, $1 \%$ EDTA solution. The epidermal layer of the dermis was then gently separated and the remaining dermis massaged to extrude the microvascular EC. The resultant cell suspension was centrifuged at $1,000 \mathrm{rpm}$ for $5 \mathrm{~min}$ and resuspended in EC culture medium consisting of Iscove's modified Dulbecco's medium (IMDM), $200 \mathrm{U} / \mathrm{ml}$ penicillin, $200 \mu \mathrm{g} / \mathrm{ml}$ streptomycin, $20 \mu \mathrm{g} / \mathrm{ml}$ gentamycin, 2 $\mathrm{mM}$ glutamine, $5 \times 10^{-5} \mathrm{M}$ 2-mercaptoethanol, $5 \times 10^{-4} \mathrm{M}$ dibutyryl 3'5'-cyclic adenosine monophosphate (Sigma Chemical Co., St. Louis, MO), $3.3 \times 10^{-5} \mathrm{M}$ isobutyl-methylxanthine (Sigma Chemical Co.) and $10 \%$ human peripartum serum. The suspended cells were then plated onto 35-mm plastic dishes (Miles Scientific, Naperville, IL) that had been coated with $2 \%$ gelatin. The cultures were expanded when confluence was achieved and utilized for functional assays within the fifth passage. Adherent cells were identified as EC by their light microscopic characteristic morphology and growth pattern, as well as the presence of Factor VIII associated protein by indirect immunofluorescence (IF). EC cultures that had $>1 \%$ contamination with other cell types (melanocytes, fibroblasts, and pericytes) were not used for functional studies.

cence-activated cell sorter; IF, immunofluorescence; IFN- $\gamma$, gamma interferon; IL-1, interleukin 1; IMDM, Iscove's modified Dulbecco's medium; LFA, lymphocyte function-associated antigen; LGL, large granular lymphocytes; mAb, monoclonal antibodies; NK, natural killer; rIL-1- $\alpha$, recombinant alpha interleukin 1; PBMC, peripheral blood mononuclear cells. 
Monoclonal antibodies. Murine monoclonal antibodies (mAb) antiLeu2a, Leu3a, Leu4, Leu7, Leu8, Leu11a, Leu11b, Leu12, and LeuM3 were obtained from the Becton Dickinson Monoclonal Center, Inc., Mountain View, CA. Anti-Leu2a (clone SK-1, IgG1) binds to the CD8 antigen present on suppressor/cytotoxic $T$ cells; anti-Leu3 (clone SK-3, IgG1) reacts with the CD4 antigen present on helper/inducer T cells; anti-Leu4 (clone SK-7, IgG1) reacts with the CD3 complex present on mature T cells; anti-Leu5b (clone S5.2) reacts with the sheep erythrocyte rosette receptor on T cells; anti-Leu7 (clone HNK-1, IgM) reacts with a subset of NK cells and a subset of $T$ cells expressing the CD3 antigen (13); anti-Leu8 (clone SK-11, IgG2a) reacts with the majority of T cells and with a subset of B cells, monocytes and granulocytes; anti-Leu 1 la (clone NP-15, IgG1) and Leu 11 b (clone GO-22, IgM) react with the Fc gamma receptor present on NK cells and granulocytes (13); anti-Leu 12 (clone 4G7, IgG1) reacts with mature B cells; anti-LeuM3 (clone MMA, $\mathrm{IgG}_{2 \mathrm{~b}}$ ) reacts with monocytes/macrophages.

Anti-LFA-1 (clone TS-122) and anti-LFA-3 antibodies, both IgG1, reacting with broadly distributed antigens present on hematopoietic, and in the case of LFA-3, hematopoietic and non-hematopoietic tissues (14), were a gift from Dr. A. Krensky at Stanford University. MAb W6/32, an IgG2a reacting with a framework specific determinant on HLA-A, B and $C$ molecules was provided by Dr. Peter Parham at Stanford University. MAb End X11 (IgG1) and HEA-14 (IgM) are endothelial cell specific antibodies produced in this laboratory (manuscript in preparation) which react with surface determinants present on human capillary and venular EC lines but not with a panel of multiple lymphoid and nonlymphoid cell lines, nor with bovine arterial EC.

Flow cytometry. IF staining was performed as described elsewhere in detail (15). All dilutions, washing and incubations were performed in cold PBS $(0.1 \mathrm{M}$ phosphate, $\mathrm{pH} 7.3)$ containing $0.1 \% \mathrm{NaN}_{3}$. Cytofluorographic analysis was performed by means of a fluorescence activated cell sorter (FACS), Ortho Cytofluorograf System 50H, using fluorochrome-conjugated mAb.

Isolation of lymphocyte subpopulations. Buffy coats from randomly selected healthy donors were obtained from the Stanford Blood Center, Stanford, CA. Peripheral blood mononuclear cells (PBMC) were isolated by Ficoll-Hypaque gradient centrifugation. Sheep erythrocyte rosetting cells (E-RFC) were separated by a single-step rosetting method (16) using neuraminidase-treated sheep erythrocytes. Rosetted cells were $>96 \%$ positive with the anti-Leu $5 \mathrm{~b} \mathrm{mAb}$ and $<0.3 \%$ positive with anti-LeuM3 when analyzed by FACS.

Lymphocyte subsets were obtained by a panning technique (17) allowing the separation of freshly isolated E-RFC into Leu2+, Leu3+, Leu8+, and Leu $11+$ subpopulations by using the respective antibodies. Subset purity was determined by indirect IF staining using the mAb with which the cells were panned. By these parameters, all subsets had a purity of $>90 \%$.

To obtain large granular lymphocyte (LGL)-enriched populations, which are also enriched in functional and phenotypic NK cells (18), monocytes were removed from PBMC by adherence $\left(60 \mathrm{~min}\right.$ at $\left.37^{\circ} \mathrm{C}\right)$ to plastic tissue culture flasks. Nonadherent cells were fractionated according to their density on discontinuous gradients of Percoll (19), followed by an additional adherence step on the LGL-enriched fraction to remove residual monocytes. This yielded a substantial enrichment of Leu $11+$ cells, from an average of $10 \%$ in the unfractionated population, to a range of $30-70 \%$ in the enriched population. There were consistently less than 5\% monocytes as determined by FACS analysis using the antiLeuM3 mAb.

Lymphocyte-endothelial cell binding assay. Binding of the various lymphocyte subpopulations to microvascular EC in culture was evaluated in a 90-min assay by adding ${ }^{51} \mathrm{Cr}$-labeled lymphocytes to confluent monolayers of EC. Briefly, microvascular EC (within the fifth passage in culture) were grown to confluence $\left(4 \times 10^{4}\right.$ cells/well) in 48-well, $2 \%$ gelatin-coated plates. Immediately before the assay, monolayers were washed once to remove detached cells. Lymphocytes were labeled with $5 \mu \mathrm{Ci}{ }^{51} \mathrm{Cr} / 10^{6}$ cells and graded numbers added to each well in a final volume of $300 \mu \mathrm{l}$ IMDM with $5 \%$ peripartum serum. After a 90 -min incubation at $37^{\circ} \mathrm{C}$, the unbound cells were removed by carefully washing the monolayers four times with warm medium using a finely tapered pasteur pipette, to allow for minimal disturbance of the monolayers. An additional wash was performed when nonadherent cells were still present after the described procedure. All wells were inspected at this point and, with rare exception, the monolayers remained intact. If there was appreciable EC detachment, these replicates were excluded from the assay. After washing, $200 \mu \mathrm{l}$ of detergent ( $0.7 \%$ Triton X-100 in glycerol-water) were added to each well and incubated for $20 \mathrm{~min}$ at $37^{\circ} \mathrm{C} .175 \mu \mathrm{l}$ of lysate were recovered and analyzed in a gamma counter (Micromedic Systems, Horsham, PA). Maximum ${ }^{51} \mathrm{Cr}$ release for each lymphocyte subpopulation and effector number was determined by incubating the same number of labeled lymphocytes in separate tubes and lysing them at the end of the incubation time as indicated above. The mean percentage of adherent lymphocytes in four replicates was then calculated as follows: $\mathrm{cpm}$ in $0.175 \mathrm{ml}$ lysate from EC monolayers/cpm in $0.175 \mathrm{ml}$ of original lymphocyte suspension $\times 100$.

Variability among the replicates was consistently $<15 \%$. In control experiments, both the adherence of E-RFC to plastic and the EC uptake of ${ }^{51} \mathrm{Cr}$ spontaneously released by labeled lymphocytes during the assay were evaluated and found negligible (data not shown).

To perform some experiments in medium depleted of immunoglobulins (Ig), IgG was adsorbed by protein A sepharose CL-4B (Pharmacia Fine Chemicals, Piscataway, NJ) affinity chromatography of peripartum human serum. This method has been shown to reliably deplete greater than $95 \%$ of $\mathrm{IgG}$ from serum (20). $\mathrm{IgG}_{3}$ binds poorly to protein $\mathrm{A}$, but comprises a minor proportion (1-3\%) of IgG in human serum. The serum used in these experiments was $98 \%$ IgG depleted as demonstrated by rate nephelometry. EC monolayers were cultured in medium containing this IgG-depleted serum prior to the assay, which itself was performed in this medium. EC cultured in human serum can be consistently shown to bear considerable surface Ig when harvested and analyzed by indirect IF and FACS analysis with a fluorescein-labeled goat anti-human Ig reagent (Zymed, So. San Francisco, CA). However, after $72 \mathrm{~h}$ culture in Ig-depleted media, there was no detectable EC surface Ig (not shown).

In some experiments the EC monolayers were treated for $4 \mathrm{~h}$ with $3 \mathrm{U} /$ well $(10 \mathrm{U} / \mathrm{ml})$ of either $\alpha$ - or $\beta$-human recombinant interleukin 1 (IL-1) (generously provided by S. Gillis of Immunex Corp., Seattle, WA) before the addition of labeled cells. The monolayers were then washed before the addition of lymphocytes, thereby removing any remaining extracellular IL-1.

To remove lymphocytes adherent to EC monolayers for further characterization, monolayers were treated after the binding assay with a $0.5 \%$ EDTA solution in $\mathrm{Ca}^{+2} \mathrm{Mg}^{+2}$-free PBS for 10 min at $37^{\circ} \mathrm{C}$, followed by repeated washings of the recovered adherent cells in RPMI $16405 \%$ fetal calf serum. Although EDTA removes EC from plastic, EC-bound lymphocytes were liberated more quickly, allowing some degree of differential recovery. However, collection of some EC could not be avoided.

Antibody inhibition studies. Blocking experiments were performed by treating either E-RFC or EC monolayers with various $\mathrm{mAb}$ prior to the binding assay. ${ }^{51} \mathrm{Cr}$-labeled E-RFC were treated with the indicated amounts of $\mathrm{mAb}$ for $30 \mathrm{~min}$ at room temperature, followed by two washes in IMDM plus $10 \%$ pooled human serum. MAb treatment of the EC was performed by incubating the monolayers with the indicated amount of antibody for $30 \mathrm{~min}$ at $37^{\circ} \mathrm{C}$, followed by two washes with warm IMDM $10 \%$ pooled human serum.

NK cytotoxicity assay. NK activity was evaluated by means of a 4$h{ }^{51} \mathrm{Cr}$ release cytotoxicity assay utilizing either the $\mathrm{K} 562$ erythroleukemia line or microvascular EC lines isolated from different individuals as targets. The indicated lymphocyte subpopulations were used as effectors. When the $\mathrm{K} 562$ line was used as a target, 5,000 ${ }^{51} \mathrm{Cr}$-labeled cells were added to each well of a round-bottom microtiter plate (Linbro-Flow Lab. Inc., McLean, VA) containing different numbers of effectors in a final volume of $200 \mu \mathrm{l}$ of IMDM plus $10 \%$ FCS. When the EC were used as targets, confluent monolayers of EC cultured in standard media or media containing Ig-depleted serum (see above) in flat-bottom microtiter plates (Linbro-Flow Lab. Inc.) were labeled with $2 \mu \mathrm{Ci} /$ well of ${ }^{51} \mathrm{Cr}$ for $90 \mathrm{~min}$ at $37^{\circ} \mathrm{C}$. After incubation, the monolayers were washed three 
times with warm medium and various numbers of effector cells were added to each well in a final volume of $200 \mu \mathrm{l}$ of culture medium. After the addition of effectors, the plates were centrifuged at $500 \mathrm{rpm}$ for 5 min at RT. Because of the well described enhancement of NK activity by IL-2 (21), the effector cells were incubated for $18 \mathrm{~h}$ at $37^{\circ} \mathrm{C}$ in culture medium with or without $50 \mathrm{U} / \mathrm{ml}$ recombinant IL-2 (Cetus Corp., Emeryville, CA) before their use in the NK assay in some experiments. In cold target inhibition experiments, the indicated numbers of unlabeled $\mathrm{K} 562$ cells were added to wells containing ${ }^{51} \mathrm{Cr}$-labeled EC monolayers before the addition of effectors. Spontaneous and maximum ${ }^{51} \mathrm{Cr}$ release were determined by incubating labeled targets in culture medium or $0.7 \%$ Triton X-100 in glycerol-water, respectively. Spontaneous release for each target never exceeded $20 \%$ of the maximum release. Mean corrected percent lysis in triplicate cultures was calculated as follows: \% cytotoxicity $=$ mean experimental $\mathrm{cpm}-$ spontaneous release $\mathrm{cpm} /$ maximum release $\mathrm{cpm}-$ spontaneous release $\mathrm{cpm} \times 100$. Replicate variability was always $<10 \%$.

\section{Results}

Basal lymphocyte-EC binding. To determine the nature and specificity of the basal binding of E-RFC to EC, freshly isolated, chromium-51-labeled E-RFC were incubated with confluent EC monolayers at varying ratios of E-RFC/EC. At E-RFC/EC ratios $>5: 1$, the percent adherence began to fall, suggesting a saturation of potential binding sites at the 5:1 ratio (Fig. 1). After an initial 90-min incubation, nonadherent lymphocytes (NAL) were recovered and reincubated with fresh EC monolayers syngeneic to the initial monolayer, in an attempt to determine whether these initially nonadherent cells were truly less efficient at binding. An $85 \%$ reduction in binding efficiency was consistently noted with the NAL population, suggesting specificity in the interaction between AL and EC. The bound lymphocytes appeared dark and slightly irregular (Fig. 2), a characteristic morphology of cells firmly adherent to EC monolayers.

Of note is that the basal binding efficiency displayed a substantial degree of variability, ranging from 8 to $31 \%$, depending

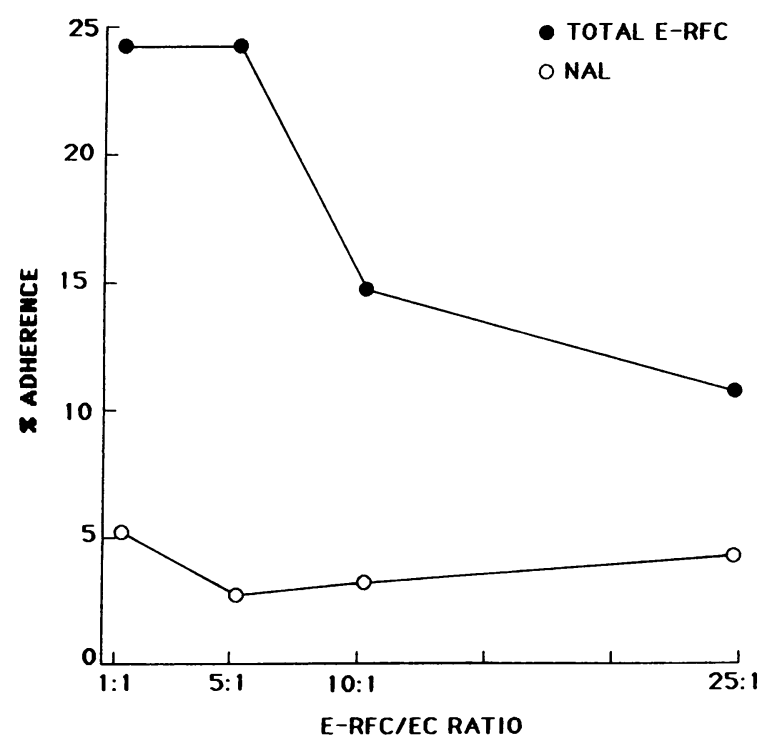

Figure 1. Specificity of lymphocyte binding to EC. Unfractionated ERFC (•) were incubated for 90 min with confluent EC monolayers, following which NAL (O) were recovered, washed, and incubated with fresh EC for an additional $90 \mathrm{~min}$. A saturation phenomenon is demonstrated by increasing the ratio of E-RFC to EC. Similar results were obtained in five of five experiments. upon the effector-target combination. There do appear to be EC lines that consistently serve as efficient binding targets when used with E-RFC from multiple unrelated donors. These results suggest the existence of intrinsic EC properties that vary the ability of lymphocytes to bind to a given EC line. In spite of this basal variability, the NAL always bound much less efficiently than unfractionated E-RFC when tested with fresh EC.

Cold lymphocyte competition. To determine whether the ECbound E-RFC could be competitively displaced, unlabeled ERFC were coincubated at varying ratios with ${ }^{51} \mathrm{Cr}$-labeled cells in the binding assay described above. The percent adherence fell sharply with unlabeled E-RFC competition, and continued to fall at a 100 unlabeled to 1 labeled cell ratio (Fig. 3), suggesting competition for specific binding sites.

Phenotype of adherent and nonadherent lymphocytes. To compare the phenotype of adherent, nonadherent, and total ERFC populations, cell sorter analysis was carried out on cells recovered before and after the binding incubation. As shown in Table I, there was a marked increase in the percentage of Leu7+ and Leu 1 + cells in the AL population, together with a marked depletion of both subsets in the NAL population. The adherent lymphocyte population also demonstrated a moderate increase in the percentage of $\mathrm{CD} 8+$ cells, and substantial depletion of CD4+ cells, resulting in a reversal of the usual ratio of CD8: CD4 cells. The Leu 2 antibody staining pattern of adherent CD8 cells differed from that of fresh E-RFC. The adherent CD8+ population showed an increase in the relative number of cells expressing a low cell surface density of Leu2, and a decrease in the number expressing a high cell surface density of Leu2 (Fig. 4). This result is consistent with the increased percentage of CD8 cells in the adherent population reflecting preferential binding of Leu 11+, "Leu2 dull" lymphocytes. Also of interest is the reduction (61\%) of Leu8+ cells in the AL population.

Binding assay using purified subsets of lymphocytes. In an attempt to confirm the results of phenotyping experiments, lymphocyte subsets were isolated by the panning technique and utilized in the EC binding assay. Leu $11+$ cells demonstrated a threefold increase in binding efficiency over the total E-RFC population, with 73.8 percent of the Leu $11+$ cells added binding to EC monolayers (Fig. 5). Leu2+ cells bound more efficiently than Leu3+ cells. Leu8 + cells bound the least efficiently, correlating well with the low percentage of Leu8+ cells found in the adherent population following culture of unfractionated E-RFC with EC (Table 1). Microscopic visualization of the monolayers after the 90-min subset binding (Fig. 2) correlated well with the quantitative assessment.

To investigate binding by cells of the natural killer phenotype isolated without either a sheep erythrocyte rosetting or an antibody selection step (in an attempt to avoid any possible modification of cell function, including activation of NK cells by Fc receptor binding), populations enriched in large granular lymphocytes (LGL) were isolated by a single step Percoll fractionation. As shown in Fig. 5, cells in the LGL-enriched fraction, which were at least threefold enriched for Leu $11+$ cells, bound three times more efficiently than the cells outside the LGL fraction.

Binding assay in immunoglobulin-free media. To examine the possibility that Leu $11+$ cells bound to EC via a nonspecific interaction between NK cell Fc gamma receptors and EC cytophilic Ig, both EC culture and the binding assay were performed in medium containing IgG-depleted serum. EC cultured in this medium did not bear detectable surface Ig, but nonetheless were 

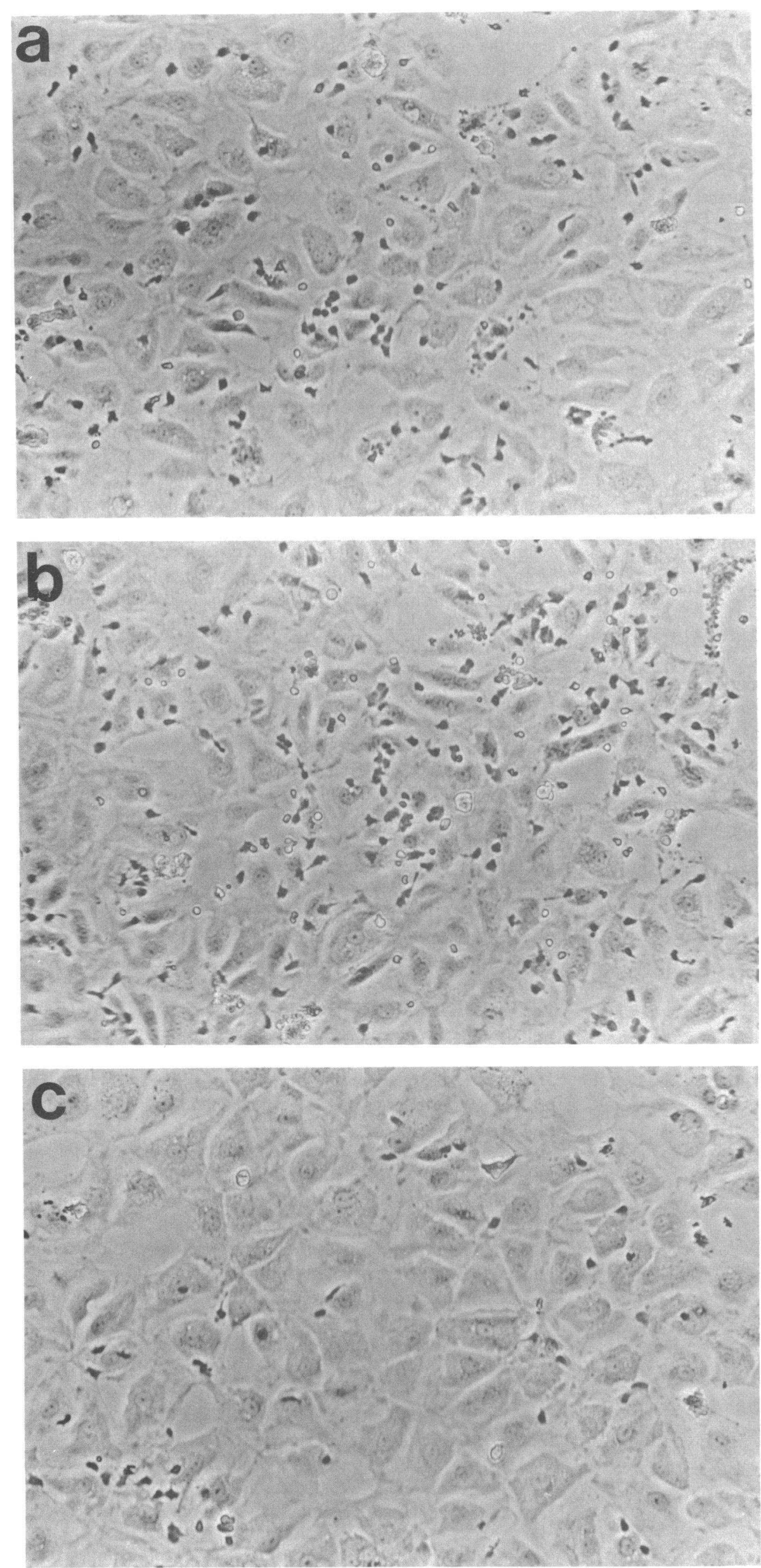

Figure 2. Phase contrast photomicrographs of lymphocyte subsets bound to EC monolayers. E-RFC and lymphocyte subsets isolated by the panning method were incubated with EC for $90 \mathrm{~min}$, after which the monolayers were extensively washed to remove nonadherent cells. The bound lymphocytes appear dark and irregular, a characteristic morphology of cells firmly adherent to EC monolayers. Differential binding efficiency is demonstrated, with total ERFC (a) binding with intermediate efficiency. Leu $11+$ cells $(b)$ bound with increased efficiency relative to the total ERFC population, whereas Leu3+ cells (c) bound relatively poorly (340X). 


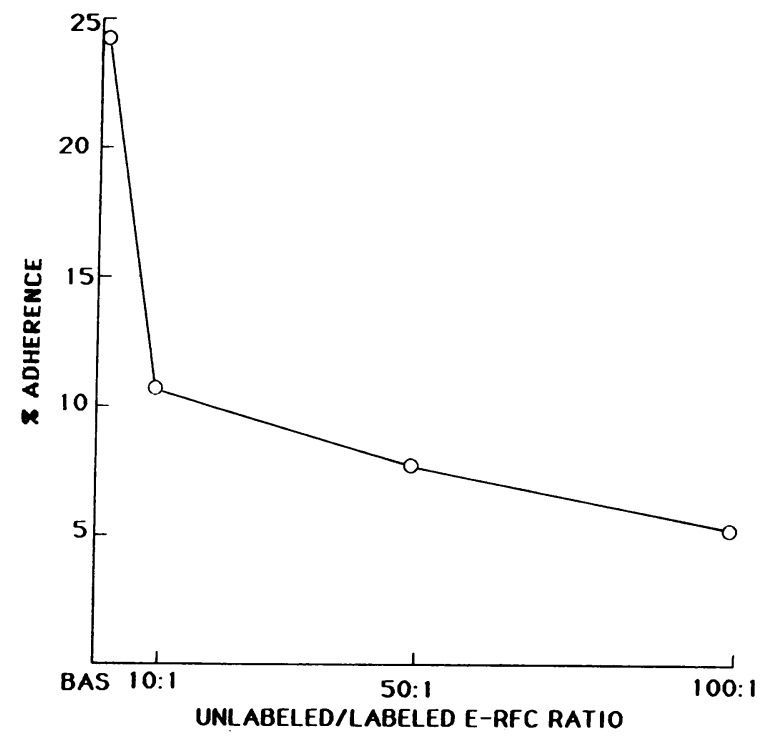

Figure 3. Competition between labeled and unlabeled E-RFC for binding to EC. Unlabeled E-RFC were added in varying ratios to ${ }^{51} \mathrm{Cr}$ labeled E-RFC obtained from the same donor, and the two populations incubated for 90 min with confluent EC monolayers. Binding efficiency was compared to the basal binding in the absence of unlabeled cells (BAS). Results are expressed as percent adherence of the labeled E-RFC to EC monolayers. Similar results were obtained in five of five experiments.

bound by E-RFC to a similar extent as EC cultured in IgGcontaining medium (Table II). Moreover, there was marked enhancement of binding by Leu $11+$ cells, relative to total E-RFC (Table II). Fetal calf serum was also used to control for the potential presence of human Ig on the EC surface. Binding results were comparable in the presence of FCS as well (data not shown).

Effect of lymphocyte pretreatment with monoclonal antibodies on lymphocyte-EC binding. To study the role of various lymphocyte surface molecules in lymphocyte binding to EC, E-RFC were treated with a panel of monoclonal antibodies and washed extensively before their incubation with EC monolayers. In all cases, $10 \mu \mathrm{g} / 10^{6}$ cells was shown to be a saturating antibody concentration and was, therefore, utilized. Although several antibodies were slightly inhibitory, including anti-Leu7 and anti-

Table I. Phenotype of EC Adherent and Nonadherent E-RFC*

\begin{tabular}{llccc}
\hline mAb & $\begin{array}{l}\text { Target } \\
\text { molecule }\end{array}$ & $\begin{array}{l}\text { E-RFC } \\
\text { \% POS }\end{array}$ & $\begin{array}{l}\text { NAL } \\
\text { \% POS }\end{array}$ & $\begin{array}{l}\text { AL } \\
\text { \% POS }\end{array}$ \\
\hline Leu2 & CD8 & 28.6 & 28.4 & 38.2 \\
Leu3 & CD4 & 52.5 & 62.9 & 9.8 \\
Leu4 & CD3 & 93.4 & 93.3 & 82.4 \\
Leu5 & CD2 & 96.4 & ND & ND \\
Leu7 & Leu7 & 10.7 & 4.1 & 31.8 \\
Leu8 & Leu8 & 60.1 & 72.3 & 23.6 \\
Leu11a & CD16 & 7.1 & 1.5 & 35.4 \\
\hline
\end{tabular}

* NAL and AL were recovered after a 90 -min binding assay. Percent of the indicated cell population (total E-RFC, NAL, or AL) were detected as positive by indirect IF and FACS analysis using the various mAb noted.
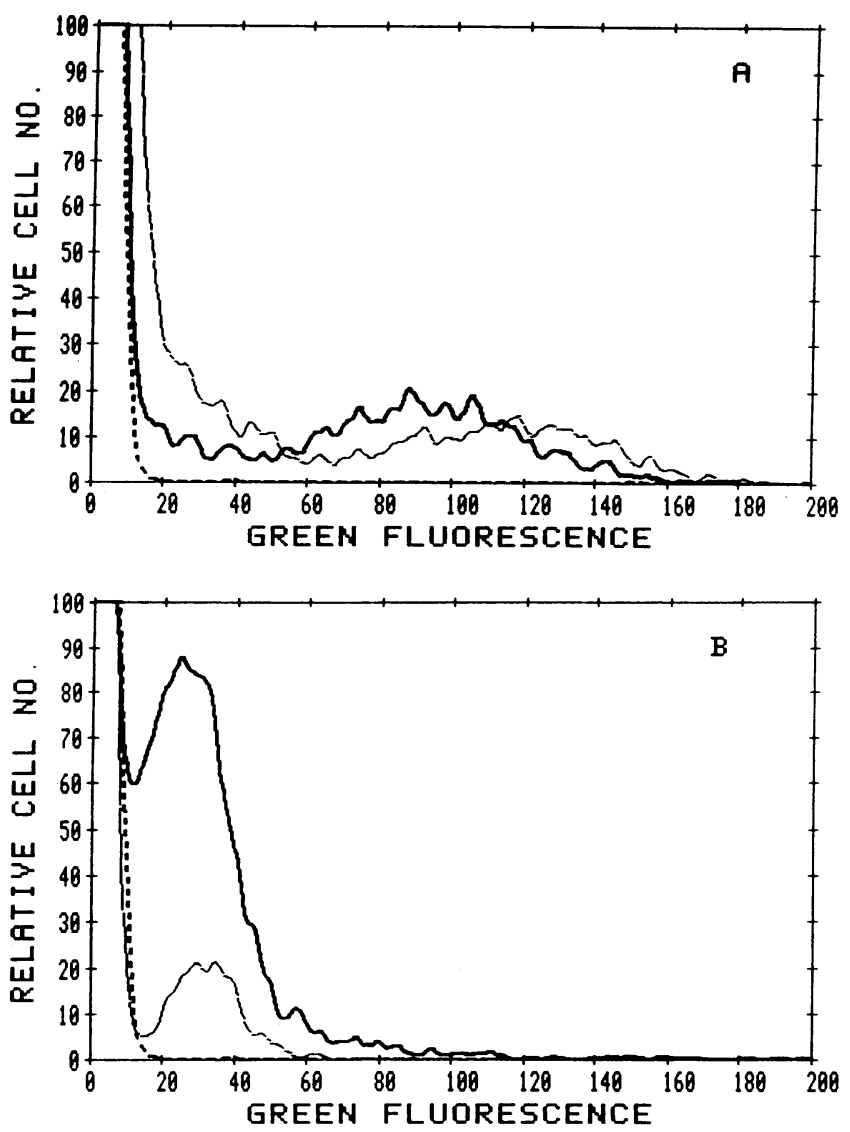

Figure 4. Cell sorter analysis of unfractionated E-RFC and AL. Unfractionated E-RFC ( - ) and $\mathrm{AL}(-)$ recovered after a 90-min incubation with confluent EC monolayers were analyzed for the presence of $(A)$ Leu2 and $(B)$ Leu3 antigens by indirect IF staining using mAb to Leu2a and Leu3a, as described in Methods. 10,000 cells were analyzed with each antibody. Irrelevant control (background) staining is also shown (- - ).

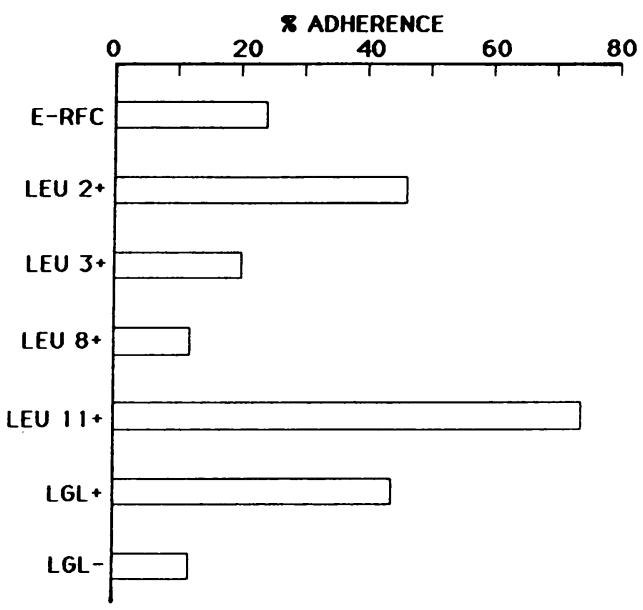

Figure 5. Lymphocyte subset binding to EC. Lymphocyte subsets isolated by the panning method were incubated with confluent EC monolayers for $90 \mathrm{~min}$ and percent adherence measured. LGL+ cells are monocyte-depleted PBLs settling at the $43.5 \%$ interface in discontinuous percoll gradient fractionation. LGL-cells comprise the pelleted fraction. Results are representative of five separate experiments. 
Table II. Binding and Cytotoxicity Assays in Immunoglobulin-depleted Media*

\begin{tabular}{|c|c|c|c|c|}
\hline & \multicolumn{2}{|c|}{ Human serum } & \multicolumn{2}{|c|}{ Ig-depleted human serum } \\
\hline & $\begin{array}{l}\% \\
\text { Binding }\end{array}$ & $\begin{array}{l}\% \\
\text { Cytotoxicity }\end{array}$ & $\begin{array}{l}\% \\
\text { Binding }\end{array}$ & Cytotoxicity \\
\hline Total E-RFC & 8.9 & ND & 14.1 & ND \\
\hline Leu $11+$ & 54.0 & 20.4 & 63.6 & 22.7 \\
\hline
\end{tabular}

* Total E-RFC and Leu 11+ cells from the same donor isolated by the panning technique were incubated for 90 min with confluent monolayers of EC which had been cultured in $10 \%$ human serum or $10 \%$ Ig-depleted human serum. The NK cytotoxicity assay was performed after overnight incubation of the effectors with $50 \mathrm{U} / \mathrm{ml} \mathrm{rIL-2} \mathrm{utilizing}$ an effector to target ratio of 50;1. The EC cultured in Ig-depleted serum had no detectable surface Ig by FACS analysis.

Leu 1 la, only anti-LFA1 provided a consistently strong degree of inhibition, notably $79 \%$ in the example shown (Fig. $6 \mathrm{~A}$ ).

Effect of EC pretreatment with monoclonal antibodies on lymphocyte-EC binding. To investigate the role of various EC surface molecules as receptors in the lymphocyte-EC adhesion process, saturating concentrations of a panel of monoclonal antibodies were used to treat the EC monolayers before incubation with E-RFC. Significant inhibition of binding was not detected with any antibodies tested, as compared with irrelevant (control)
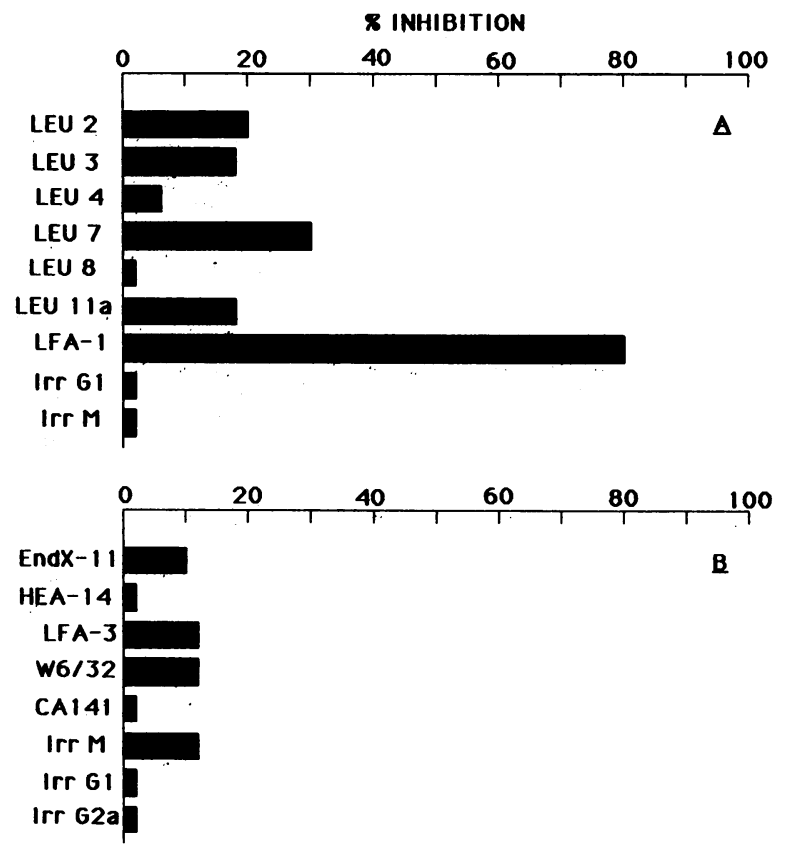

Figure 6. Effects of selected monoclonal antibodies on E-RFC-EC binding. $(A) \mathrm{E}-\mathrm{RFC}$ or $(B) \mathrm{EC}$ were incubated with saturating concentrations of designated $\mathrm{mAbs}$ and washed prior to a 90-min incubation of the E-RFC with the confluent EC monolayers. Irrelevant (IRR) antibodies are isotype matched with test reagents and have demonstrated absence of detectable binding to either $(A)$ E-RFC or $(B)$ EC, by indirect IF and FACS analysis. Binding after $\mathrm{mAb}$ treatment is compared with basal, unmodified binding and data expressed as percent inhibition of basal adherence. Results are representative of three separate experiments. monoclonal antibodies (Fig. 6 B). Two EC-specific murine monoclonal antibodies generated in our laboratory, designated End X11 and HEA-14, also failed to inhibit binding.

Effect of EC treatment with IL-1 on lymphocyte-EC binding. IL-1 has recently been shown to enhance lymphocyte binding to umbilical vein EC (10). To determine whether IL-1 treatment of EC affected lymphocyte binding to microvascular EC, the same subset binding experiment described above was performed utilizing EC monolayers pretreated with either recombinant alpha or beta IL-1 at $10 \mathrm{U} / \mathrm{ml}$. Both cytokines enhanced adherence in all subsets, including those that bound inefficiently in the absence of rIL-1 (Table III). This is most dramatically represented with the Leu8 + subset, which as shown above, bound poorly to EC in the absence of exogenous IL-1.

Ability of $L G L$-enriched populations to lyse EC. To assess the ability of Percoll gradient-enriched LGL to kill EC, and to compare binding and killing efficiency, untreated LGL-enriched cells or those incubated overnight with $50 \mathrm{U} / \mathrm{ml}$ of rIL-2 were incubated with EC monolayers derived from three unrelated donors, and cytolysis was determined. Standard NK assays with classical targets (K562 cells) were simultaneously performed to assess NK activity in the conventional system. Two EC lines, numbers 30 and 33, were bound by a high percentage (31.5 and $29 \%$, respectively) of PBMC, whereas line 44 was bound with intermediate efficiency (Table IV). LGL-enriched cells consistently bound EC more efficiently than PBMC, whereas LGLdepleted cells adhered less well (Fig. 5). IL-2 had no effect on binding efficiency of any population (not shown). Of the two lines bound by a high percentage of lymphocytes, one served as a relatively sensitive target for NK activity and one did not. The EC line that was bound least well was killed very weakly. These findings demonstrate that EC can serve as NK targets but the ability of EC to bind LGL correlates poorly with their susceptibility to NK-mediated cytolysis. As shown in the same table, overnight incubation of effectors with $50 \mathrm{U} / \mathrm{ml} \mathrm{rIL-2,} \mathrm{which} \mathrm{is}$ known to enhance NK activity against other targets (21), enhanced the killing of all three EC targets as well as the K562 line. The degree of rIL-2 enhancement of EC killing correlated with the level of basal killing. However, none of the EC lines were as sensitive to NK mediated lysis as K562 cells.

To determine whether EC cytotoxicity was mediated by the same cells which are responsible for classical NK activity, cold target inhibition experiments were performed by adding increasing numbers of unlabeled $\mathrm{K} 562$ cells to ${ }^{51} \mathrm{Cr}$-labeled EC monolayers before the addition of Leu $11+$ effector cells isolated

Table III. Binding of Isolated Lymphocyte Subsets to Untreated and IL-1-treated EC

\begin{tabular}{llll}
\hline Subset* & Basal & $\alpha \mathrm{IL}-1 \quad 10 \mathrm{U} / \mathrm{ml}$ & $\beta \mathrm{IL}-1 \quad 10 \mathrm{U} / \mathrm{ml}$ \\
\hline Total E-RFC & $24.0 \%$ & $42.9 \%$ & $41.9 \%$ \\
Leu2+ & 44.1 & 63.7 & 52.8 \\
Leu3+ & 21.6 & 53.1 & 53.7 \\
Leu8+ & 11.1 & 35.4 & 38.0 \\
Leu11+ & 72.4 & 93.4 & 86.3
\end{tabular}

\footnotetext{
* Various lymphocyte subsets were positively selected by the panning technique using the indicated $\mathrm{mAb}$. Control basal binding in this experiment was preceded by a 4-h EC incubation in media alone. Data expressed as percent adherence to EC.
} 
Table IV. NK Activity of PBL, LGL-enriched and LGL-depleted Cells, Against K562 Cells and Three Different EC Lines*

\begin{tabular}{|c|c|c|c|c|c|c|c|c|}
\hline \multirow[b]{3}{*}{ Effectors } & \multicolumn{8}{|l|}{ Targets } \\
\hline & \multicolumn{2}{|l|}{ K562 } & \multicolumn{2}{|c|}{$\begin{array}{l}\text { EC } 30 \\
\text { (Adherence of PBL 32\%) }\end{array}$} & \multicolumn{2}{|c|}{$\begin{array}{l}\text { EC } 33 \\
\text { (Adherence of PBL 29\%) }\end{array}$} & \multicolumn{2}{|c|}{$\begin{array}{l}\text { EC } 44 \\
\text { (Adherence of PBL 19\%) }\end{array}$} \\
\hline & $50: 1$ & $25: 1$ & $50: 1$ & $25: 1$ & $50: 1$ & $25: 1$ & $50: 1$ & $25: 1$ \\
\hline PBL $^{\ddagger}$ & $36.8 \%$ & $27.3 \%$ & $10.3 \%$ & $9.5 \%$ & $7.2 \%$ & $4.2 \%$ & $2.3 \%$ & $1.5 \%$ \\
\hline $\mathrm{PBL}+\mathrm{IL}-2^{\S}$ & 57.6 & 40.5 & 16.8 & 9.1 & 10.1 & 5.4 & 10.4 & 8.1 \\
\hline LGL enriched" & 61.8 & 51.7 & 13.7 & 6.3 & 6.5 & 0 & 3.7 & 1.5 \\
\hline LGL enriched + IL-2 & 82.0 & 80.0 & 35.0 & 15.3 & 13.8 & 2.0 & 7.2 & 2.8 \\
\hline LGL depleted' & 4.4 & 2.6 & 2.9 & 0.7 & 0 & 0 & 1.2 & 0 \\
\hline LGL depleted + IL-2 & 7.0 & 6.7 & 3.7 & 0.4 & ND & ND & ND & ND \\
\hline
\end{tabular}

* The various effector cells were incubated with the indicated ${ }^{51} \mathrm{Cr}$-labeled targets in a 4-h cytotoxicity assay. Binding assays were also performed utilizing PBL and the three EC target lines. 50:1 and 25:1 indicate effector to target ratios tested.

${ }^{\ddagger}$ Monocyte-depleted PBL.

\$ Monocyte-depleted PBL treated overnight with $50 \mathrm{U} / \mathrm{ml}$ rIL-2.

"LGL-enriched cells are monocyte-depleted PBLs settling at the $43.5 \%$ interface in discontinuous Percoll gradient fractionation.

' LGL-depleted cells comprise the pelleted fraction.

by the panning technique. Basal Leu $11+$ cell-mediated cytotoxicity was $39 \%$. At an unlabeled $\mathrm{K} 562$ to ${ }^{51} \mathrm{Cr}$-labeled EC ratio of 0.5 to 1 , a $92 \%$ reduction in cytotoxicity was demonstrated.

To examine whether NK-mediated cytolysis of EC occurred via a nonspecific, Fc receptor-mediated mechanism (antibody dependent cellular cytotoxicity), Leu $11+$ cells were utilized as effectors in parallel killing assays performed with targets cultured in human serum and Ig-depleted serum. NK activity against EC was comparable with the two media tested (Table II).

NK activity of ALs against K562 cells and EC. To compare the capacity of AL and NAL to mediate NK activity, AL and NAL were recovered after incubation with EC monolayers and tested for the ability to lyse conventional (K562 cells) and EC
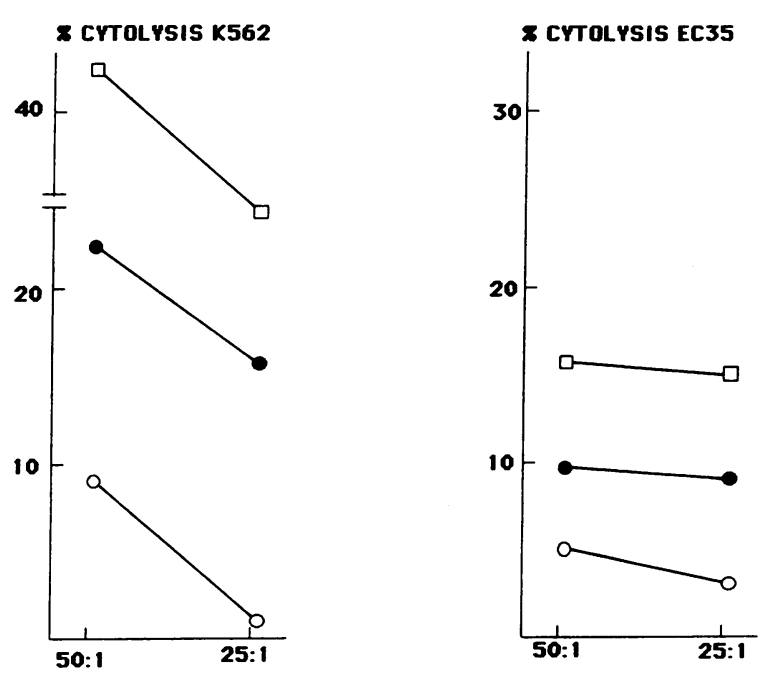

Figure 7. NK activity of AL and NAL. E-RFC were incubated for 90 min with confluent monolayers of EC line 35 . AL were recovered by gentle EDTA treatment and used as effectors in a 4-h cytotoxicity assay, with the relevant EC35 line, and K562 cells employed as targets. Cytolytic activity of $\mathrm{AL}(\square)$ were compared with that of unfractionated E-RFC (๑) and NAL (O). Similar results were demonstrated in four of four experiments. targets. There was an enhancement in NK activity of the recovered AL as compared with total E-RFC, and a marked depletion of NK activity in the NAL (Fig. 7). This was observed with both K562 and EC targets.

\section{Discussion}

There is considerable evidence in humans and animals that the endothelium is a critical target in the rejection of vascularized allografts $(7,22)$. More specifically, the published data implicate the microvasculature in this role, with the envelopment of capillaries, venules, and arterioles by lymphocytes apparently mediating early microvascular endothelial damage. Most studies utilizing EC as in vitro targets have used umbilical vein as an EC source. In the current study, we have employed microvascular EC since such cells are likely to be more physiologically relevant targets.

Conjugate formation between potential effector cells and targets is generally accepted as a necessary early event in various cell-mediated immune responses. This binding is probably not antigen-specific $(23,24)$ but may contribute to the priming of $T$ cells in the afferent limb of the immune response. The binding assay employed in this study allows sufficient quantitation to evaluate both the kinetics and specificity of binding. Other previously described techniques employed for evaluating mononuclear cell-EC binding are much less quantitative $(25,26)$, making evaluation of binding parameters impossible. In this study, we utilized competition experiments and generation of saturation by radiolabeled cells to demonstrate that binding of E-RFC to EC is mediated via a receptor-ligand interaction. At ratios greater than five lymphocytes to one $\mathrm{EC}$, the percentage of the total cells binding to the monolayers consistently fell, suggesting saturation of binding above this ratio. The relative inefficiency of binding by recovered NAL also strongly suggests that this interaction is specific.

A striking finding in these experiments was the preferential binding of Leu 1 $1+$ NK cells to microvascular EC. Past studies of these cells have largely centered on their interaction with transformed or virally infected cells (27). There is relatively little 
known regarding the interaction between natural killer cells and biologically normal, nonlymphoid targets. The preferential binding of Leu 1 + cells to EC was demonstrated by phenotyping the adherent and nonadherent cells (Table I), as well as by the isolated subset binding experiment (Table III). NAL were nearly depleted of Leu $11+$ cells, and the majority of positively selected Leul $1+$ cells bound to the EC monolayers. This binding does not appear related to "activation" of Leu $11+$ cells by sheep cell rosetting or Leu 11 antibody treatment, as LGL-enriched cells isolated by Percoll density fractionation demonstrated the same enhancement in binding (Fig. 5). Because EC cultured in human serum bear surface Ig, the possibility that binding of Leu11+ cells occurs nonspecifically via Fc receptors was addressed. Pretreatment of lymphocytes with a saturating concentration of antiLeu 1 la, which interacts with NK cell Fc receptors, and is known to block NK cell Fc receptor-mediated binding (28), failed to inhibit binding (Fig. 6). Also, Leu11+ cells were isolated by antibody treatment of cells with anti-Leu11c, panning, and overnight incubation at $37^{\circ} \mathrm{C}$ before use in the binding assay. We and others (28) have demonstrated phenotypic and functional modulation of the NK cell Fc receptor upon antibody treatment and 18-h culture. Most importantly, when EC were cultured in IgG-depleted medium and observed to be surface Ig negative before the binding assay, Leu11+ cells displayed the same degree of binding efficiency when compared with binding utilizing surface Ig-positive EC as targets (Table II).

In contrast to the recent finding that CD4 $T$ cells bind preferentially to gamma interferon treated, $D R+$ umbilical vein endothelial cells (25), there was a marked depletion of CD4+ cells in our adherent cell population. This is not surprising since our EC targets were DR - (data not shown). On the other hand, it is probable that the increased percentage of CD8 cells in the population adherent to EC reflects the preferential binding to EC by CD8+,Leu 11 + NK cells. Leu 11+, "CD8 dull” lymphocytes have been demonstrated as a small subset in the peripheral blood, which contains the majority of mononuclear cells capable of NK activity (29).

Treatment of the lymphocytes with anti-LFA-1 antibodies markedly inhibited binding to EC. The LFA-1 molecule is a heterodimer composed of noncovalently associated $\alpha$ - and $\beta$ polypeptide chains of 177,000 and $95,000 \mathrm{~mol} \mathrm{wt}$, respectively. It is present in varying density on $B$ and $T$ cells and has been shown to be important in a variety of leukocyte functions dependent on target adhesion, including conjugate formation between cytolytic $T$ lymphocytes and their targets $(23,30,31)$. A role for LFA-1 in leukocyte adhesion to $(32,33)$ and cytolytic T lymphocyte-mediated cytotoxicity against (34) umbilical vein EC has been shown. Antibodies to the LFA-1 molecule have also been demonstrated to inhibit killing by NK clones and peripheral blood NK cells $(35,36)$, and lymphocytes isolated from LFA-1 deficient patients demonstrate defective NK activity (37). Our results indicate that the LFA-1 molecule also plays an essential role in the binding of NK cells to microvascular EC. This does not appear to be based upon a "like-like" LFA-1 receptorligand interaction, as LFA-1 is absent from the resting, microvascular EC surface (data not shown). Although LFA-3 is highly expressed on the EC surface (data not shown), antibodies to this molecule also did not prevent binding, making it unlikely that LFA-1-LFA-3 or CD2-LFA-3 (38) interactions play an essential role in NK-EC adherence. Antibodies to a variety of additional lymphocyte and EC surface molecules had no effect on lymphocyte-EC binding (Fig. 6), suggesting that these molecules do not play a role in the adherence of these cell types. However, the involvement of these various molecules cannot be completely dismissed, as it remains possible that the mAb used did not recognize the relevant molecular epitope.

Our results do not demonstrate that lymphocyte-EC binding is a function of allogeneic recognition. The possibility exists that autologous binding of lymphocytes, including Leu $11+$ cells, to EC can occur. In fact, cord blood lymphocytes have been shown to bind equally well to syngeneic and allogeneic IFN-treated umbilical vein EC (25). Also, homotypic adhesion of lymphocytes exposed to various stimuli has been demonstrated, with involvement of the LFA-1 molecule (26). Thus, accessory molecules clearly play a general role in cellular conjugate formation $(23,24)$. However, binding events between autologous cells mediated entirely by accessory molecules may not provide a sufficient stimulus to promote mutual cellular activation, or trigger further immunologic events. Additional studies will be necessary to address this question.

The EC monolayers used in this study were susceptible to NK-mediated cytolysis despite the fact that these cells are neither transformed nor virally infected. This killing did not appear to be mediated by Fc receptors (Table II) and was competitively inhibited by classical NK targets. Interestingly, the degree of sensitivity to natural killing varied between EC isolates. EC lines sensitive to one allogeneic effector were generally sensitive to multiple different allogeneic effectors, whereas EC lines relatively resistant to natural killing consistently demonstrated that feature in response to multiple allogeneic effectors (data not shown). The variability in EC susceptibility to NK mediated cytolysis was not a function of the age of the EC monolayers, as all targets were used for functional studies during the fifth passage. In addition, although EC varied in their capacity to bind lymphocytes, efficient binding did not ensure efficient killing (Table IV). Thus, there were several instances in which a high degree of adherence was associated with poor killing. On the other hand, efficient killing in the presence of inefficient binding was not seen, supporting the concept that adhesion is required but not sufficient to induce cytolysis. As has been shown with other targets (21), IL-2 enhanced NK mediated cytolysis against all targets tested. However, this lymphokine had no effect on adherence (not shown).

In contrast to the selective binding by Leu $11+$ cells to untreated EC, a high percentage of all lymphocyte subsets bound EC which had been precultured in rIL-1. In our view, the lack of specificity of the rIL-1 effect does not diminish its potential importance, since such an effect might promote extravascular lymphocyte mobilization in vivo and, thereby, augment an ongoing immune response. Moreover, although NK cells appear to have some capacity to lyse microvascular EC in vitro, such killing does not necessarily contribute to allograft rejection. Rather, binding of EC by NK cells may trigger a specific, HLAdirected cellular immune response, resulting in rejection. NK cells and EC have both been shown to produce IL-1 (39-43). Thus, adhesion could promote a mutual activation of both EC and NK cells, with production of IL-1 as well as IFN- $\gamma$ (44). Once IFN- $\gamma$ is produced in sufficient quantities and DR antigen is induced on the EC surface, a secondary wave of a classic HLA reactive cellular events could follow, further triggered by the previously documented enhanced binding of $T$ cells to IFN- $\gamma$ exposed EC $(11,25)$. In this regard, recent studies of a rat renal allograft model reveal an early peak of LGL infiltrating grafts at day 4. By day 6, negligible numbers of LGL are in the graft, 
and by day $7, T$ cells accumulate in association with extensive tissue destruction. On the other hand, the possibility remains that EC restricted determinants may be target molecules in acute rejection of vascularized allografts. Further studies will be necessary to define these antigens and their role.

\section{Acknowledgments}

We thank Donna Jones for preparation of this manuscript. We are grateful to Dennis T. Sasaki and Stephanie Dumas for their assistance with cytofluorography. We also thank Leslie Tackett and Jane Eaton for their technical assistance with endothelial cell culture.

This work was supported by grant HL-13108 from the National Institutes of Health and the Joseph Drown Foundation.

\section{References}

1. Loveland, B. E., and I. F. C. McKenzie. 1982. Which T cells cause graft rejection? Transplantation. 33:217-221.

2. Loveland, B. E., P. M. Hogarth, R. Ceredig, and I. F. C. McKenzie. 1981. Cells mediating graft rejection in the mouse. J. Exp. Med. 153: 1044-1049.

3. Nemlander, A., E. Saksela, and P. Hayry. 1983. Are "natural killer" cells involved in allograft rejection? Eur. J. Immunol. 13:348-350.

4. Brasile, L., J. Clarke, T. Galouzis, and J. Cerilli. 1985. The clinical significance of the vascular endothelial cell antigen system: evidence for genetic linkage between the endothelial cell antigen system and the major histocompatibility complex. Transplant. Proc. 17:741-743.

5. Daar, A. S., S. V. Fuggle, J. W. Faber, A. Ting, and P. J. Morris. 1984. The detailed distribution of HLA-A,B,C antigens in normal human organs. Transplantation. 38:287-292.

6. Daar, A. S., S. V. Fuggle, J. W. Faber, A. Ting, and P. J. Morris. 1984. The detailed distribution of MHC class II antigens in normal human organs. Transplantation. 38:293-297.

7. Forbes, R. D. C., R. D. Guttman, M. Gomersall, and J. Hibberd. 1983. A controlled serial ultrastructural tracer study of first-set cardiac allograft rejection in the rat: evidence that the microvascular endothelium is the primary target of graft destruction. Am. J. Pathol. 111:184-190.

8. Chin, Y. H., G. D. Carey, and J. J. Woodruff. 1982. Lymphocyte recognition of lymph node high endothelium. IV. Cell surface structures mediating entry into lymph nodes. J. Immunol. 129:1911-1915.

9. Gallartin, W. M., I. L. Weissman, and E. C. Butcher. 1983. A cell-surface molecule involved in organ-specific homing of lymphocytes. Nature (Lond.). 304:30-34.

10. Cavender, D. E., D. O. Haskard, B. Joseph, and M. Ziff. 1986. Interleukin 1 increases the binding of human $B$ and $T$ lymphocytes to endothelial cell monolayers. J. Immunol. 136:203-207.

11. Yu, C.-L., D. O. Haskard, D. Cavender, A. R. Johnson, and M. Ziff. 1985. Human gamma interferon increases the binding of $T$ lymphocytes to endothelial cells. Clin. Exp. Immunol. 62:554-560.

12. Davison, P. M., K. Bensch, and M. A. Karasek. 1980. Isolation and growth of endothelial cells from the microvessels of the newborn human foreskin in cell culture. J. Invest. Dermatol. 75:316-320.

13. Lanier, L. L., A. Mile, J. H. Phillips, N. L. Warner, and G. F. Babcock. 1983. Subpopulations of human natural killer cells defined by expression of the Leu 7 (HNK-1) and Leu 11 (NKP-15) antigens. J. Immunol. 131:1789-1794.

14. Sanchez-Madrid, F., A. M. Krensky, C. F. Ware, E. Robbins, J. Strominger, S. J. Burakoff, and T. A. Springer. 1982. Three distinct antigens associated with human T-lymphocyte-mediated cytolysis: LFA1, LFA-2, and LFA-3. Proc. Natl. Acad. Sci. USA. 79:7489-7493.

15. Lifson, J., A. Raubitschek, C. Benike, K. Koths, A. Ammann, P. Sondel, and E. G. Engleman. 1986. Purified interleukin 2 induces proliferation of fresh human lymphocytes in the absence of exogenous stimuli. J. Biol. Response Mod. 5:61-72.

16. Weiner, M. S., C. Bianco, and V. Nussenzweig. 1973. Enhanced binding of neuraminidase-treated sheep erythrocytes to human $\mathrm{T}$ lymphocytes. Blood. 42:939-945.

17. Wysocki, L. J., and V. L. Sato. 1978. Panning for lymphocytes. A method of cell separation. Proc. Natl. Acad. Sci. USA. 75:2844-2847.

18. Timonen, T., J. R. Ortaldo, and R. B. Herberman. 1982. Analysis by a single cell cytotoxicity assay of natural killer (NK) cell frequencies among human large granular lymphocytes and of the effects of interferon on their activity. J. Immunol. 128:2514-2522.

19. Timonen, T., and E. Saksela. 1980. Isolation of human natural killer cells by density gradient centrifugation. J. Immunol. Methods. 36: 285-298.

20. Goding, J. W. 1978. Use of staphylococcal protein A as an immunological reagent. J. Immunol. Methods. 20:241-253.

21. Lanier, L. L., C. J. Benike, J. H. Phillips, and E. G. Engleman. 1985. Recombinant interleukin 2 enhanced natural killer cell-mediated cytotoxicity in human lymphocyte subpopulations expressing the Leu 7 and Leu 11 antigens. J. Immunol. 134:794-801.

22. Dvorak, H. F., M. C. Mihm, A. M. Dvorak, B. A. Barnes, E. J. Manseau, and S. J. Galli. 1979. Rejection of first-set skin allograft in man. The microvasculature is the critical target of the immune response. J. Exp. Med. 150:322-337.

23. Spits, H., W. Van Schooten, H. Keizer, G. Van Seventer, M. Van De Rijn, C. Terhorst, and J. E. Devries. 1986. Alloantigen recognition is preceded by nonspecific adhesion of cytotoxic $\mathrm{T}$ cells and target cells. Science (Wash. DC). 232:403-405.

24. Yanelli, J. R., J. A. Sullivan, G. L. Mandell, and V. H. Engelhard. 1986. Reorientation and fusion of cytotoxic $T$ lymphocyte granules after interaction with target cells as determined by high resolution cinemicrography. J. Immunol. 136:377-382.

25. Masuyama, J., N. Minato, and S. Kano. 1986. Mechanisms of lymphocyte adhesion to human vascular endothelial cells in culture. $T$ lymphocyte adhesion to endothelial cells through endothelial HLA-DR antigens induced by gamma interferon. J. Clin. Invest. 77:1596-1605.

26. DeBono, D. 1978. Endothelium-lymphocyte interaction in vitro: II. Adherence of allergized lymphocytes. Cell. Immunol. 44:64-70.

27. Herberman, R. B. 1980. Natural Cell-mediated Immunity Against Tumors. Academic Press, New York.

28. Perussia, B., G. Trinchieri, A. Jackson, N. C. Warner, J. Faust, H. Rumpold, D. Kraft, and L. L. Lanier. 1984. The Fc receptor for IgG on human natural killer cells: phenotypic, functional, and comparative studies with monoclonal antibodies. J. Immunol. 133:180-189.

29. Lanier, L. L., E. G. Engleman, P. Gatenby, G. F. Babcock, N. L. Warner, and L. A. Herzenberg. 1983. Correlation of functional properties of human lymphoid cell subsets and surface marker phenotypes using multiparameter analysis and flow cytometry. Immunol. Rev. 74: 143-160.

30. Krensky, A. M., E. Robbins, T. A. Springer, and S. J. Burakoff. 1984. LFA-1, LFA-2, and LFA-3 antigens are involved in CTL-target conjugation. J. Immunol. 132:2180-2182.

31. Davignon, D., E. Martz, T. Reynolds, K. Kurzinger, and T. A. Springer. 1981. Monoclonal antibody to a novel lymphocyte functionassociated antigen (LFA-1): mechanism of blocking of $T$ lymphocytemediated killing and effects on other $\mathrm{T}$ and $\mathrm{B}$ lymphocyte functions. $J$. Immunol. 127:590-598.

32. Haskard, D., D. Cavender, P. Beatty, T. Springer, and M. Ziff. 1986. T lymphocyte adhesion to endothelial cells: Mechanisms demonstrated by anti-LFA-1 monoclonal antibodies. J. Immunol. 137:29012906.

33. Pohlman, T. H., K. A. Stanness, P. G. Beatty, H. D. Ochs, and J. H. Harlan. 1986. An endothelial cell surface factor(s) induced in vitro by lipopolysaccharide, interleukin 1 , and tumor necrosis factor alpha increases neutrophil adherence by a CDw18-dependent mechanism. $J$. Immunol. 136:4548-4557.

34. Collins, T., A. M. Krensky, C. Clayberger, W. Fiers, M. A. Gimbrone, Jr., S. J. Burakoff, and J. S. Pober. 1984. Human cytolytic T lymphocyte interactions with vascular endothelium and fibroblasts: Role of effector and target cell molecules. J. Immunol. 133:1878-1884.

35. Phillips, J. H., and G. F. Babcock. 1983. NKP-15: A monoclonal 
antibody reactive against purified human natural killer cells and granulocytes. Immunol. Lett. 6:143-149.

36. Schmidt, R. E., G. Bartley, H. Levine, S. F. Schlossman, and J. Ritz. 1985. Functional characterization of LFA-1 antigens in the interaction of human NK clones and target cells. J. Immunol. 135:10201025.

37. Kohl, S., T. A. Springer, F. C. Schmalstieg, L. S. Loo, and D. C. Anderson. 1984. Defective natural killer cytotoxicity and polymorphonuclear leukocyte antibody dependent cellular cytotoxicity in patients with LFA-1/OKM-1 deficiency. J. Immunol. 133:2972-2978.

38. Shaw, S., G. E. Ginther Luce, R. Quinones, R. E. Gress, T. A. Springer, and M. E. Sanders. 1986. Two antigen-independent adhesion pathways used by human cytolytic T-cell clones. Nature (Lond.). 233: 262-264.

39. Scala, G., P. Allavena, J. V. Djeu, T. Kasahara, J. R. Ortaldo, R. B. Herberman, and J. J. Oppenheim. 1984. Human large granular lymphocytes are potent producers of interleukin 1. Nature (Lond.). 309: 56-59.
40. Miossec, P., D. Cavender, and M. Ziff. 1986. Production of interleukin 1 by human endothelial cells. J. Immunol. 136:2486-2491.

41. Wagner, C. R., R. M. Vetto, and D. R. Burger. 1985. Expression of I-region-associated antigen (Ia) and interleukin 1 by subcultured human endothelial cells. Cell. Immunol. 93:91-104.

42. Libby, P., J. M. Ordovos, K. R. Auger, A. H. Robbins, L. K. Birinyi, C. A. Dinarello. 1986. Endotoxin and tumor necrosis factor induce interleukin-1 gene expression in adult human vascular endothelial cells. Am. J. Pathol. 124:179-185.

43. Stern, D. M., I. Bank, P. P. Nawroth, J. Cassimeris, W. Kisiel, J. W. Fenton II, C. Dinarello, L. Chess, and E. A. Jaffe. 1985. Selfregulation of procoagulant events on the endothelial cell surface. J. Exp. Med. 162:1223-1235.

44. Djeu, J. Y., N. Stocks, K. Zoon, G. J. Stanton, T. Timonen, and R. B. Herberman. 1982. Positive selfregulation of cytotoxicity in human natural killer cells by production of interferon upon exposure to influenza and herpes viruses. J. Exp. Med. 156:1222-1234. 